\title{
Transcriptome analysis of the hippocampus in environmental noise-exposed SAMP8 mice reveals regulatory pathways associated with Alzheimer's disease neuropathology
}

Donghong $\mathrm{Su}^{1+}$, Wenlong $\mathrm{Li}^{1+}$, Huimin $\mathrm{Chi}^{1,2+}$, Honglian Yang ${ }^{1}$, Xiaojun She ${ }^{1}$, Kun Wang ${ }^{1}$, Xiujie Gao ${ }^{1}$, Kefeng Ma ${ }^{1}$, Ming Zhang ${ }^{3^{*}}$ and Bo Cui ${ }^{1^{*}}$ (D)

\begin{abstract}
Background: Chronic noise exposure is one environmental hazard that is associated with genetic susceptibility factors that increase Alzheimer's disease (AD) pathogenesis. However, the comprehensive understanding of the link between chronic noise stress and AD is limited. Herein, we investigated the effects of chronic noise exposure on AD-like changes in senescence-accelerated mouse prone 8 (SAMP8).

Methods: A total of 30 male SAMP8 mice were randomly divided into the noise-exposed group, the control group, and aging group (positive controls), and mice in the exposure group were exposed to $98 \mathrm{~dB}$ SPL white noise for 30 consecutive days. Transcriptome analysis and AD-like neuropathology of hippocampus were examined by RNA sequencing and immunoblotting. Enzyme-linked immunosorbent assay and real-time PCR were used to further determine the differential gene expression and explore the underlying mechanisms of chronic noise exposure in relation to $A D$ at the genome level.
\end{abstract}

Results: Chronic noise exposure led to amyloid beta accumulation and increased the hyperphosphorylation of tau at the Ser202 and Ser404 sites in young SAMP8 mice; similar observations were noted in aging SAMP8 mice. We identified 21 protein-coding transcripts that were differentially expressed: 6 were downregulated and 15 were upregulated after chronic noise exposure; 8 genes were related to AD. QPCR results indicated that the expression of Arc, Egr1, Egr2, Fos, Nauk1, and Per2 were significantly high in the noise exposure group. These outcomes mirrored the results of the RNA sequencing data.

Conclusions: These findings further revealed that chronic noise exposure exacerbated aging-like impairment in the hippocampus of the SAMP8 mice and that the protein-coding transcripts discovered in the study may be key candidate regulators involved in environment-gene interactions.

Keywords: Environmental noise, Alzheimer's disease, RNA sequencing, SAMP8 mice

\footnotetext{
* Correspondence: iamcuib@sina.com; mingle1981@163.com

${ }^{\dagger}$ Donghong Su, Wenlong Li and Huimin Chi contributed equally to this work

${ }^{1}$ Tianjin Institute of Environmental and Operational Medicine, Tianjin, China

${ }^{3}$ Tianjin Centers for Disease Control and Prevention, Tianjin, China

Full list of author information is available at the end of the article
}

(c) The Author(s). 2020 Open Access This article is distributed under the terms of the Creative Commons Attribution 4.0 International License (http://creativecommons.org/licenses/by/4.0/), which permits unrestricted use, distribution, and reproduction in any medium, provided you give appropriate credit to the original author(s) and the source, provide a link to the Creative Commons license, and indicate if changes were made. The Creative Commons Public Domain Dedication waiver (http://creativecommons.org/publicdomain/zero/1.0/) applies to the data made available in this article, unless otherwise stated. 


\section{Introduction}

Alzheimer's disease (AD) is the most common form of dementia, accounting for $50-56 \%$ of autopsy and clinical cases [1]. It is marked by a progressive loss of memory and cognitive function, overproduction of amyloid beta $(A \beta)$, and increased hyperphosphorylation of tau $[1,2]$. However, the pathogenesis of $\mathrm{AD}$ is complex, and the disease has no clear cause.

Generally, the association among environmental stress, aging process, and their causal roles plays a role in the development of AD pathogenesis [3-5]. Aging is the major risk factor for AD [6]. The incidence of dementia increases exponentially with increasing age [7]. AD-like pathological changes, such as aggregation of $A \beta$ and the phosphorylation of tau protein, are associated with aging $[8,9]$. Noise-induced health effects were first recognized in industrial settings, such as manufacturers, where intense impulse sound or steady-state long-term exposure with sound pressure levels higher than $85 \mathrm{dBA}$ were associated with auditory and non-auditory effects [10]. Chronic noise exposure in experimental animals can cause not only accelerated $A \beta$ synthesis but also significant and persistent hyperphosphorylation of tau and the formation of prominent pathological neurofibrillary tangles (NFTs) of tau in the hippocampus, key structures in learning and memory, and initial sites of tau pathology in $\mathrm{AD}[11-14]$. On the other hand, chronic noise exposure could affect the spatial learning and memory [15, 16]. Hence, noise exposure may aggravate the development of aging-related AD-like pathological changes, and its related molecular mechanisms need to be explored further.

In the present study, we performed a comparative gene expression analysis of chronic noise-affected and nonnoise-affected brain tissues by using the RNA sequencing technique, an advanced approach that determines the differential expression profiles underlying phenotypic differences [17]. We selected senescence-accelerated mouse prone 8 (SAMP8), which shares behavioral, cognitive, and neuropathological alterations observed in AD patients and is a plausible natural model for exploring the pathogenesis of $\mathrm{AD}$ [18]. Our data will serve as a new reference for the prevention and treatment of AD.

\section{Materials and methods}

\section{Animal use and experimental grouping}

The 30 male SAMP8 mice used in our study were provided by the Tianjin University of Traditional Chinese Medicine. The mice were kept under standard housing conditions with controlled ambient temperature (23 \pm $2{ }^{\circ} \mathrm{C}$ ) and humidity (50-60\%) and a 12-h light/12-h dark cycle (lights on from 06:00 to 18:00). Food and water were provided in their home cages, and they were allowed to adapt to the laboratory environment for 5 days before carrying out the experiment.

Three-month-old male SAMP8 mice were randomly separated into the control group (control group) and noise exposure group exposed to $98 \mathrm{~dB}$ SPL white noise (noise group). Eight-month-old male SAMP8 mice were used as positive controls (aging group). The number of animals in each group is equal. The noise group was exposed to $98 \mathrm{~dB}$ SPL white noise from 8:00 to 12:00 for 30 days while the control group and the aging group housed in similar cages were exposed to background noise $(<40 \mathrm{~dB}$ SPL) from another chamber. After 30 consecutive days of exposure to noise, the mice were sacrificed, and brain samples were immediately collected for biochemical analyses and stored at $-80^{\circ} \mathrm{C}$ until use. All experiments adhered to the guidelines of the National Institute of Health for the use of experimental animals and were performed in accordance with the approved guidelines specified by the Animal and Human Use in Research Committee of the Tianjin Institute of Environmental Medicine and Operational Medicine.

\section{Noise exposure setup}

All noise exposures were performed as previously described [19]. Noise was generated using a noise generator (BK 3560C, B\&K Instruments, Denmark), amplified using a power amplifier (Yong-Sheng Audio P-150D; The Third Institute of China Electronics Technology Group, Beijing, China), and transmitted through a loudspeaker (ZM-16S; Tianjin Zenmay Electroacoustic Equipment Co., Tianjin, China). The frequency of the noise emitted from the speaker was in the range of 200$6300 \mathrm{~Hz}$ (1/3 octave bands). Animals were exposed to noise in a reverberation chamber, where wire-mesh cages were placed at the center of the sound field. A loudspeaker was suspended directly above the cages. The noise level variation was less than $2 \mathrm{~dB}$ in the available space of the animals. The background noise level in the chamber was below $40 \mathrm{~dB}$ SPL.

\section{Detection of $A \beta$ by enzyme-linked immunosorbent assay}

Frozen mouse hippocampus was homogenized in icecold $1 \times$ phosphate-buffered saline $(0.02 \mathrm{~mol} / \mathrm{L}, \mathrm{pH} 7.0-$ 7.2). $A \beta 1-40$ and $A \beta 1-42$ concentrations were measured in soluble and insoluble brain fractions with mouse ELISA kits (BlueGene Biotech, Shanghai, China) in accordance with the manufacturer's instructions. The mean value of the duplicate samples was considered as the final concentration for each animal.

\section{Western blot analysis}

Hippocampus preparation and Western blot were performed as described previously [13, 20]. Briefly, each hippocampus was dissected immediately after sacrificing 
the animal and was stored at $-80{ }^{\circ} \mathrm{C}$ until use. For immunoblot analysis, the frozen hippocampi were homogenized in ice-cold $50 \mathrm{mM}$ Tris- $\mathrm{HCl}$ buffer (pH 7.4) containing $1 \%$ Triton X-100, $0.2 \mathrm{mM}$ PMSF, and $1 \mathrm{mM}$ EDTA. Homogenates were centrifuged at $12,000 \times g$ for $10 \mathrm{~min}$ at $4{ }^{\circ} \mathrm{C}$. The supernatants obtained were immediately placed in boiling water for $10 \mathrm{~min}$. Samples $(10 \mu \mathrm{g}$ protein/lane) were separated on 10\% SDS-PAGE gels and electrophoretically transferred to nitrocellulose membranes. The membranes were probed with rabbit antibodies against the following proteins: Tau (C-17) (polyclonal, 1:1000, Bioworld Technology, China), PS202 (polyclonal, 1:1000, Bioworld Technology, China), PS404 (polyclonal, 1:1000, Bioworld Technology, China), Egr1 (polyclonal, 1:1000, Bioworld Technology, China), and cFos (polyclonal, 1:1000, Bioworld Technology, China). Anti-GAPDH (1:10,000; Bioworld Technology) was used as the internal reference standard.

\section{RNA quantification and qualification}

RNA degradation and contamination were monitored using $1 \%$ agarose gel. RNA purity was checked using the NanoPhotometer ${ }^{\circ}$ spectrophotometer (IMPLEN, CA, USA). RNA concentration was measured using the Qubit $^{\circ}$ RNA assay kit in Qubit $^{\circ} 2.0$ Fluorometer (Life Technologies, CA, USA). RNA integrity was assessed using the RNA Nano 6000 assay kit of the Bioanalyzer 2100 system (Agilent Technologies, CA, USA).

\section{Library preparation for transcriptome sequencing}

For the RNA sample preparations, $3 \mu \mathrm{g}$ RNA per sample was used as input material. Sequencing libraries were generated using NEBNext ${ }^{\circ}$ UltraTM RNA library prep kit for Illumina ${ }^{\circ}$ (NEB, USA), and index codes were added to attribute sequences to each sample. Briefly, mRNA was purified from total RNA by using poly-T oligo-attached magnetic beads. Fragmentation was carried out using divalent cations under elevated temperature in NEBNext first-strand synthesis reaction buffer $(5 \times)$. Clustering was performed on a cBot cluster generation system by using TruSeq PE cluster kit v3cBot-HS (Illumina). The RNA integrity number (RIN) of all samples was greater than 6.8 .

\section{Read mapping to the reference genome}

Reference genome and gene model annotation files were directly downloaded from the genome website. The index of the reference genome was built using Hisat2 v2.0.5, and paired-end clean reads were aligned to the reference genome by using Hisat2 v2.0.5 (ftp://ftp. ensembl.org/pub/release-94/gtf/mus_musculus/). We selected Hisat2 as the mapping tool to allow Hisat2 to generate a database of splice junctions on the basis of the gene model annotation file and obtain better mapping results than those obtained by other non-splice mapping tools.

\section{Quantification of gene expression level}

FeatureCounts v1.5.0-p3 was used to count the read numbers mapped to each gene. Then, the fragments per kilobase of transcript sequence per million (FPKM) of each gene were calculated on the basis of the length of the gene and the number of reads mapped to this gene. The method of using the expected number of FPKM base pairs sequenced considers the effects of sequencing depth and gene length on the read count and is currently the most commonly used technique for estimating gene expression levels. The correlations between samples were evaluated using Pearson's correlation coefficient (Additional file 1: Figure S1, A). And the principal component analysis (PCA) was used to assess the intergroup differences and the repetition of the samples within the group (Additional file 1: Figure S1, B).

\section{Differential expression analysis}

Differential expression analysis of two conditions/groups (two biological replicates per condition) was performed using the DESeq2 $\mathrm{R}$ package (1.16.1). DESeq2 provides statistical routines for determining the differential expression in the digital gene expression data by using a model based on a negative binomial distribution. The resulting $P$ values were adjusted using the Benjamini and Hochberg's approach for controlling false discovery rates. Genes with an adjusted $P$ value $<0.05$ found by DESeq2 were regarded as differentially expressed.

\section{Gene Ontology and Kyoto Encyclopedia of Genes and Genomes enrichment analysis of differentially expressed genes}

GO enrichment analysis of differentially expressed genes was implemented using the cluster Profiler R package, in which gene length bias was corrected. GO terms with corrected $P$ value $<0.05$ were considered significantly enriched by differentially expressed genes. KEGG is a database resource for understanding the high-level functions and utilities of the biological system. We used the cluster Profiler $\mathrm{R}$ package to test the statistical enrichment of the differentially expressed genes in the KEGG pathways. Environmental noise-related genes and pathways were analyzed by Gene Ontology (GO) and Pathway-Relation-Network (Path-net) analysis tools based on Kyoto Encyclopedia of Genes and Genomes (KEGG) Pathway Database using Gene Cloud of Biotechnology Information (GCBI Platform, Shanghai, China) (www.gcbi.com.cn). 
Determination of gene expression by real-time PCR

Real-time PCR was performed as previously described [20]. The hippocampus tissues from the exposure and control group mice were homogenized using a rapidly oscillating masher. Total RNA was extracted using an RNeasy Mini kit (TaKaRa Bio, Dalian, China) according to the manufacturer's protocol. Total RNA was converted to cDNA via reverse transcription by using a transcriptor first-strand cDNA synthesis kit (TaKaRa Bio, Dalian, China). A primer pair designed to amplify the GAPDH gene was used as an internal control. Specific primers and probes designed for mouse Arc, Egr1, Egr2, c-Fos, Hmgcr, Hap1, Nauk1, Per2, and GAPDH were used as described in Table 1. Gene expression levels were assessed by quantitative real-time PCR under the following thermal cycling conditions: $2 \mathrm{~min}$ at $50{ }^{\circ} \mathrm{C}$ and $10 \mathrm{~min}$ at $95^{\circ} \mathrm{C}$ followed by 45 cycles of $95^{\circ} \mathrm{C}$ for $5 \mathrm{~s}$ and $57^{\circ} \mathrm{C}$ for $30 \mathrm{~s}$. Real-time PCR was performed using gene expression assays-on-demand and a Takara PCR thermal cycler dice real-time system (TaKaRa Bio, Dalian, China). The threshold cycle $(\mathrm{Ct})$ of the target genes was normalized to that of GAPDH. mRNA levels in noiseexposed animals were calculated after normalizing the cycle thresholds to the GAPDH expression and were presented as fold-induction values $\left(2^{-\Delta \Delta C t}\right)$ relative to the control mice.

\section{Statistics}

Results were expressed as mean \pm standard deviation of the mean. Data were analyzed using the SPSS v.19.0 software (SPSS Inc., Chicago, IL, USA). Data were subjected to one-way analysis of variance, followed by the least significant difference method test or Dunnett's $t$ test. Values were considered to be significantly different at $P<0.05$.

Table 1 Mouse primers used for quantitative real-time PCR

\begin{tabular}{|c|c|}
\hline Gene & Primers \\
\hline Egr2 & $\begin{array}{l}\text { F:5'-GGAGAGAGTCAGTGACGGATAGA-3' } \\
\text { R:5'-TTTGCTCCTCGCACAACCTG-3' }\end{array}$ \\
\hline Egr1 & $\begin{array}{l}\text { F:5'-CCACCATGGACAACTACCCC-3' } \\
\text { R:5'-TCATAGGGTTGTCGCTCGG-3' }\end{array}$ \\
\hline Fos & $\begin{array}{l}\text { F:5'-TACTACCATTCCCCAGCCGA-3' } \\
\text { R:5'-GCTGTCACCGTGGGGATAAA-3' }\end{array}$ \\
\hline Hap1 & $\begin{array}{l}\text { F:5'-GCCCATCTAGAAACCCCAGC-3' } \\
\text { R:5'-AGGGCCATGAAGACGAAAGG-3' }\end{array}$ \\
\hline Nauk1 & $\begin{array}{l}\text { F:5'-GGACGAGCTAGACATGGTTCA-3' } \\
\text { R:5'-AGTAATGCACGGCAGACACA-3 }\end{array}$ \\
\hline Per2 & $\begin{array}{l}\text { F:5'-CCACTATGTGACAGCGGAGG-3' } \\
\text { R:5'-TGTCGGGCTCTGGAATAAGC-3' }\end{array}$ \\
\hline $\mathrm{Hmgcr}$ & $\begin{array}{l}\text { F:5'-TGAGATCCGGAGGATCCAAGG-3' } \\
\text { R:5'-CAGATCTTGTTGTTGCCGGTG-3' }\end{array}$ \\
\hline Arc & $\begin{array}{l}\text { F:5'-TGGAGGGAGGTCTTCTACCG-3' } \\
\text { R:5'-CCTACAGAGACAGTGTGGCG-3 }\end{array}$ \\
\hline GAPDH & $\begin{array}{l}\text { F:5'-AGGTCGGTGTGAACGGATTTG-3' } \\
\text { R:5'-TGTAGACCATGTAGTTGAGGTCA-3' }\end{array}$ \\
\hline
\end{tabular}

\section{Results}

Chronic noise exacerbates AD-like neuropathology in SAMP8 mice

To explore the effects of chronic noise exposure on $A \beta$ in the hippocampus, we determined the concentrations of soluble and insoluble $A \beta 1-40$ and $A \beta 1-42$ in the brain components by using a mouse ELISA kit. The results showed that the amount of $A \beta 1-40$ or $A \beta 1-42$ in the soluble or insoluble fractions was significantly increased in the noise group relative to the control group and was close to that of the aging group (Fig. 1a-d).

Tau phosphorylation levels were assessed by quantitative immunoblot analyses of cortical extracts from individual mouse in each group after the end of the 30-day experiment period (Fig. 1e, f). In comparison with those in the control group, the levels of tau phosphorylated at Ser202 and Ser404 in the noise group were significantly increased (Fig. 1g, h). These data suggested that chronic noise exposure accelerated AD-like pathological alterations in the SAMP8 mice.

\section{Functional enrichment analysis}

A total of $161,813,829$ raw reads $(80,365,338$ for the noise group and $81,448,491$ for the control group) were generated. After discarding the reads with adapters, poly-N > $10 \%$, and any other possible contaminants, $156,995,998$ clean reads $(78,392,394$ for the noise group and $78,603,604$ for the control group) were obtained. The clean reads were mapped to the mouse reference genome, and the mapping rates were approximately 92.93\% and $93.53 \%$ for the noise and control group mice, respectively. The cufflink results indicated 30,673 protein-coding transcripts. These mRNAs were used for subsequent analysis.

To identify significant associations of genes with any specific molecular pathway, we performed annotation enrichment analyses. Genes that significantly enriched for $P$ value in GO terms included transmembrane receptor protein serine/threonine kinase signaling pathway, synaptic transmission (GABAergic), sleep and regulation of synaptic transmission (GABAergic), and several terms were closely related to $\mathrm{AD}$, such as learning or memory (GO: 0007611 ), cognition (GO: 0050890), learning (GO: 0007612), and regulation of neuronal synaptic plasticity (GO: 0048168) (Table 2). By contrast, the KEGG results revealed 20 pathways, of which had significant $P$ values included Toll-like receptor signaling pathway, TNF signaling pathway, and MAPK signaling pathway (Glutamatergic synapse) (Table 3). We also observed that Egr1, Fos, Egr2, and Arc were the main central genes, and they directly interacted with each other in the gene network analysis. 
A

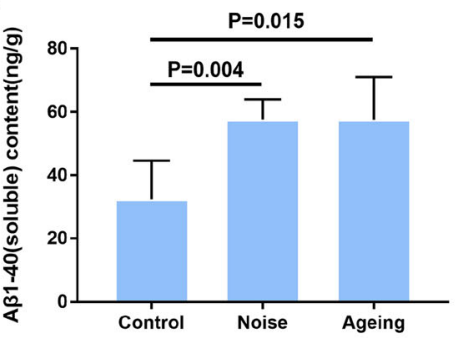

C

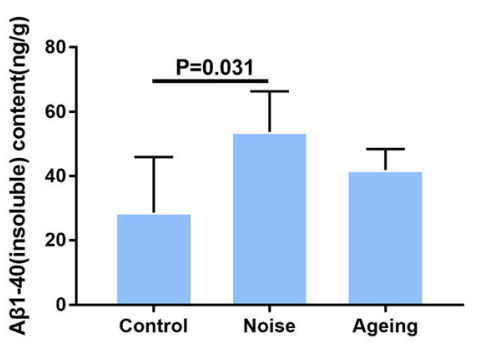

$\mathbf{E}$
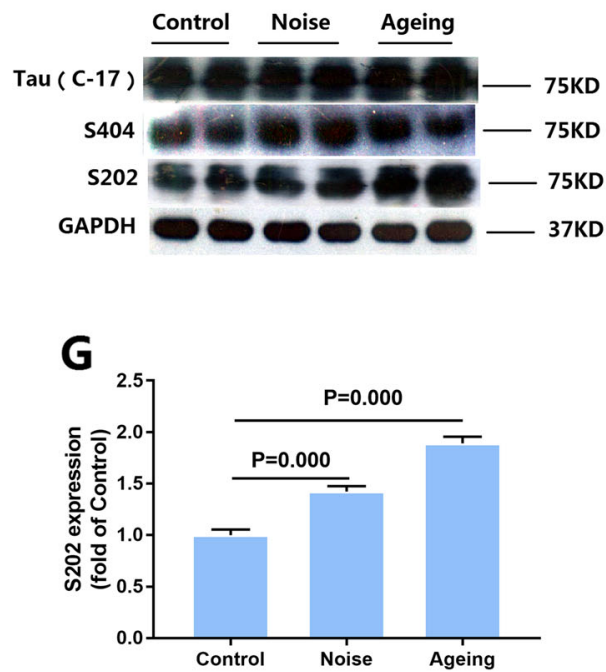

B

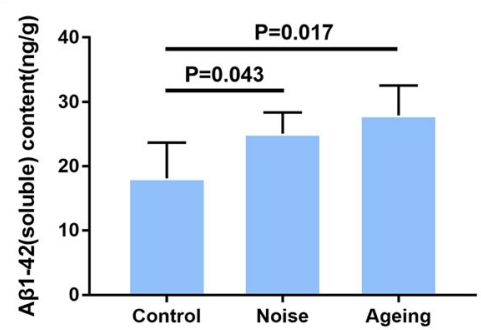

D
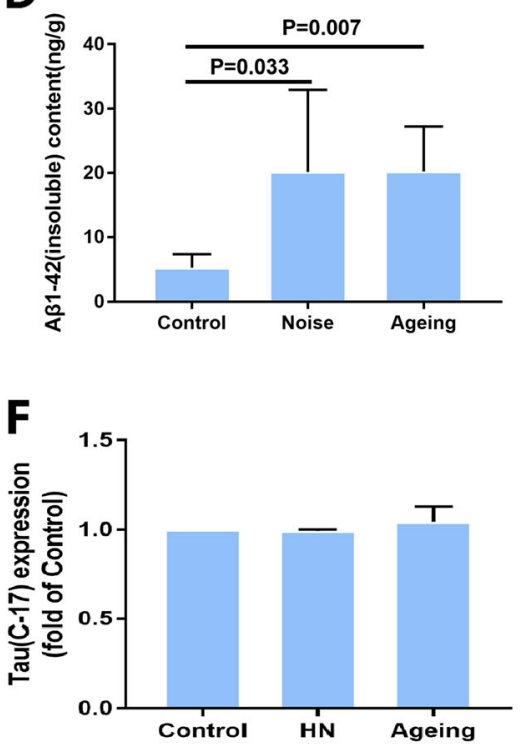

H

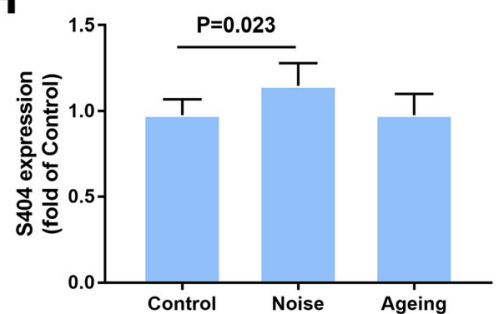

Fig. 1 Chronic noise-induced Alzheimer's disease (AD)-like pathological alterations in SAMP8 mice. a-d Quantification of soluble and insoluble A 1-40 and AB1-42 levels by ELISA. e The expression of total tau and hyperphosphorylated tau in SAMP8 mice. $\mathbf{f}-\mathbf{h}$ The density of the immunoreactive bands was quantified and represented as a percent change relative to the control. GAPDH was used as a loading control. Data were expressed as the mean \pm standard deviation ( $n=6$ per group). Results were normalized as the control $=100 \%$

\section{Differential expression analysis}

The mRNA expression levels and transcripts were estimated using FPKMs. A total of 30,673 mRNA transcripts were obtained. Expression analysis showed that 21 mRNA transcripts, including 15 upregulated and 6 downregulated transcripts $(P<0.05)$, were differentially expressed in the noise group relative to the control group (Table 4). Differentially expressed mRNAs were analyzed using the volcano map and clustering heat map (Fig. 2a, b). Exactly 8 of the 21 differentially expressed genes were related to $\mathrm{AD}$ (Table 5).
Validation and quantitative assessment of selected ADrelated differential genes

Eight AD-related differentially expressed mRNA transcripts were selected to validate the accuracy of RNA sequencing via qPCR (Fig. 3a-h). The noise-exposed groups exhibited significantly higher mRNA expression of Arc, Egr1, Egr2, Fos, Nauk1, and Per2 than the control mice, thereby confirming the results of the RNA sequencing data. The mRNA expression of Arc, Egr1, and Fos was also markedly higher in the aging mice than in the control mice; the mRNA expression in the latter was 
Table 2 Chronic noise exposure Significantly affected gene ontology

\begin{tabular}{|c|c|c|c|}
\hline Go ID & Go Description & $P$ value & Overlap gene name \\
\hline GO:0007611 & learning or memory & 0.000154 & Egr2; Fos;Hmgcr;Arc \\
\hline GO:0050890 & cognition & 0.000235 & Egr2; Fos; Hmgcr; Arc \\
\hline GO:0007612 & learning & 0.000598 & Fos; Hmgcr; Arc \\
\hline GO:0048168 & regulation of neuronal synaptic plasticity & 0.001688 & Egr2; Arc \\
\hline GO:0007215 & glutamate receptor signaling pathway & 0.003899 & Homer1; Arc \\
\hline GO:0008306 & associative learning & 0.004507 & Fos; Hmgcr \\
\hline GO:0007568 & aging & 0.004563 & Fos; Nuak1; Hmgcr \\
\hline GO:0050804 & modulation of synaptic transmission & 0.004607 & Egr2; Hap1; ArC \\
\hline GO:0045073 & regulation of chemokine biosynthetic process & 0.011953 & Egr1 \\
\hline GO:1902992 & negative regulation of amyloid precursor protein catabolic process & 0.011953 & Hap1 \\
\hline GO:0048167 & regulation of synaptic plasticity & 0.014307 & Egr2; Arc \\
\hline GO:0032230 & positive regulation of synaptic transmission, GABAergic & 0.015513 & Hap1 \\
\hline GO:0007216 & G-protein coupled glutamate receptor signaling pathway & 0.017879 & Homer 1 \\
\hline GO:1902003 & regulation of beta-amyloid formation & 0.017879 & Hap1 \\
\hline GO:1902991 & regulation of amyloid precursor protein catabolic process & 0.021418 & Hap1 \\
\hline GO:0034205 & beta-amyloid formation & 0.022595 & Hap1 \\
\hline GO:2000311 & regulation of AMPA receptor activity & 0.024945 & Arc \\
\hline GO:0032731 & positive regulation of interleukin-1 beta production & 0.030797 & Egr1 \\
\hline GO:1900271 & regulation of long-term synaptic potentiation & 0.031963 & Arc \\
\hline GO:0050435 & beta-amyloid metabolic process & 0.033128 & Hap1 \\
\hline GO:0032732 & positive regulation of interleukin-1 production & 0.034292 & Egr1 \\
\hline GO:2000772 & regulation of cellular senescence & 0.034292 & Nuak1 \\
\hline GO:0030431 & sleep & 0.035454 & Fos \\
\hline GO:0032228 & regulation of synaptic transmission, GABAergic & 0.038933 & Hap1 \\
\hline GO:0043666 & regulation of phosphoprotein phosphatase activity & 0.0424 & Nuak1 \\
\hline GO:0090342 & regulation of cell aging & 0.0424 & Nuak1 \\
\hline GO:1900449 & regulation of glutamate receptor signaling pathway & 0.0424 & Arc \\
\hline GO:1901214 & regulation of neuron death & 0.04305 & Fos; Egr1 \\
\hline GO:0007178 & transmembrane receptor protein serine/threonine kinase signaling pathway & 0.044463 & Fos; Egr1 \\
\hline GO:0042982 & amyloid precursor protein metabolic process & 0.044705 & Hap1 \\
\hline GO:0099601 & regulation of neurotransmitter receptor activity & 0.044705 & Arc \\
\hline GO:0051932 & synaptic transmission, GABAergic & 0.047004 & Hap1 \\
\hline GO:0032651 & regulation of interleukin-1 beta production & 0.048152 & Egr1 \\
\hline
\end{tabular}

similar to that in the noise-exposed mice. No betweengroup differences were observed in the mRNA expression of Hmgcr. The mRNA expression of Hapl significantly increased in the noise and aging groups, whereas it decreased in the RNA sequencing. To further validate the accuracy of the RNA sequencing, the Egr1 and Fos levels were assessed by Western blot analysis. Immunoblotting confirmed that the expressions of Egr1 and Fos increased more in the noise-exposed and aging mice than in the control mice (Fig. 3i-k). These results indicated that chronic noise exposure induced aging-like impairment in the hippocampi of the SAMP8 mice.

\section{Discussion}

Multiple factors contribute to the pathogenesis of $\mathrm{AD}$, and they include aging, genetic variables, and environmental factors [21]. Chronic noise exposure, the main focus of environmental factors, is related to AD [12-14, 22]. Acute or chronic noise exposure could induce various indicators of AD-like pathological changes that increase the risk of AD development [19, 23]. Our recent research further confirmed that chronic noise exposure led to cognitive impairment and $A \beta$ accumulation in young SAMP8 mice, similar to that observed in aging SAMP8 mice [12]. In the present study, we described a reliable transcriptome 
Table 3 Chronic noise exposure significantly affected signaling pathways

\begin{tabular}{lllll}
\hline Path ID & Path description & P value & Gene name & KEGG_ID \\
\hline mmu04727 & GABAergic synapse & 0.170713 & Hap1 & mmu:15114 \\
mmu04620 & Toll-like receptor signaling pathway & 0.172572 & Fos & mmu:14281 \\
mmu04933 & AGE-RAGE signaling pathway in diabetic complications & 0.199989 & Egr1 & mmu:13653 \\
mmu04668 & TNF signaling pathway & 0.205371 & Fos & mmu:14281 \\
mmu04724 & Glutamatergic synapse & 0.217796 & Homer1 & mmu:26556 \\
mmu04725 & Cholinergic synapse & 0.219557 & Fos & mmu:14281 \\
mmu04152 & AMPK signaling pathway & 0.231776 & Hmgcr & mmu:15357 \\
mmu04728 & Dopaminergic synapse & 0.245524 & Fos & mmu:14281 \\
mmu04024 & CAMP signaling pathway & 0.343077 & Fos & mmu:14281 \\
mmu04010 & MAPK signaling pathway & 0.476833 & Fos & mmu:14281 \\
\hline
\end{tabular}

sequencing to highlight key proteins in the hippocampus that are modulated in response to AD-like neurodegenerations. We also reported genomic signatures that are associated with chronic noise exposure and with age-related changes in the tissue.

By overlaying transcriptomic and neuropathological profiles, we identified noise and age-related shifts in underappreciated pathways, such as the Toll-like receptor (TLR) signaling pathway, TNF signaling pathway, MAP kinase pathway, and Ras/ERK signaling pathway, which are involved in the pathogenesis of AD. TLRs, a family of receptor proteins, play a wide role in innate and adaptive immune responses upon the stimulations by exogenous and endogenous TLR ligands. There is an increased endocytosis of TLR4 in the AD model mice brain, which might be a key event for the neurodegeneration signaling in the brain [24]. TNF- $\alpha$ has been assessed in the pathophysiology of AD both in human [25] and animal studies [26], and in our previous study, chronic noise exposure increased levels of TNF- $\alpha$ in the rat hippocampus [20]. The mammalian MAPK family consists of p38-MAPK, ERK, and c-Jun NH2-terminal kinase (JNK), which is

Table 4 Twenty-one significantly dysregulated mRNAs in the noise-exposed and control groups

\begin{tabular}{|c|c|c|c|}
\hline Gene & Transcript ID & Foldchange & Description \\
\hline Egr2 & ENSMUSG00000037868 & 7.249386 & early growth response 2 \\
\hline Homer1 & ENSMUSG00000007617 & 2.090347 & homer scaffolding protein 1 \\
\hline Fos12 & ENSMUSG00000029135 & 1.690786 & fos-like antigen 2 \\
\hline Fos & ENSMUSG00000021250 & 2.703889 & FBJ osteosarcoma oncogene \\
\hline Rps27a & ENSMUSG00000020460 & 0.617494 & ribosomal protein S27A \\
\hline Egr1 & ENSMUSG00000038418 & 1.877242 & early growth response 1 \\
\hline Hap1 & ENSMUSG00000006930 & 0.619126 & huntingtin-associated protein 1 \\
\hline Gfod1 & ENSMUSG00000051335 & 1.48084 & glucose-fructose oxidoreductase domain \\
\hline Slc7a1 & ENSMUSG00000041313 & 1.46453 & solute carrier family 7 \\
\hline Ankrd33b & ENSMUSG00000022237 & 1.689168 & ankyrin repeat domain 33B \\
\hline Nuak1 & ENSMUSG00000020032 & 1.485235 & NUAK family, SNF1-like kinase,1 \\
\hline Gm10076 & ENSMUSG00000060143 & 0.470904 & predicted gene 10076 \\
\hline Rn7sk & ENSMUSG00000065037 & 4.981484 & RNA, 7SK, nuclear \\
\hline Zfp366 & ENSMUSG00000050919 & 2.106077 & zinc finger protein 366 \\
\hline B2m & ENSMUSG00000060802 & 0.575968 & beta-2 microglobulin \\
\hline Per2 & ENSMUSG00000055866 & 1.838931 & period circadian clock 2 \\
\hline Tppp3 & ENSMUSG00000014846 & 0.585384 & tubulin polymerization-promoting protein \\
\hline mt-Nd41 & ENSMUSG00000065947 & 0.414951 & mitochondrially encoded NADH \\
\hline Hmger & ENSMUSG00000021670 & 1.685124 & 3-hydroxy-3-methylglutaryl-Coenzyme A \\
\hline Arc & ENSMUSG00000022602 & 1.892969 & activity regulated cytoskeletal-associated protein \\
\hline Elov16 & ENSMUSG00000041220 & 1.465985 & ELOVL family member 6 , elongation of long chain fatty acids (yeast) \\
\hline
\end{tabular}



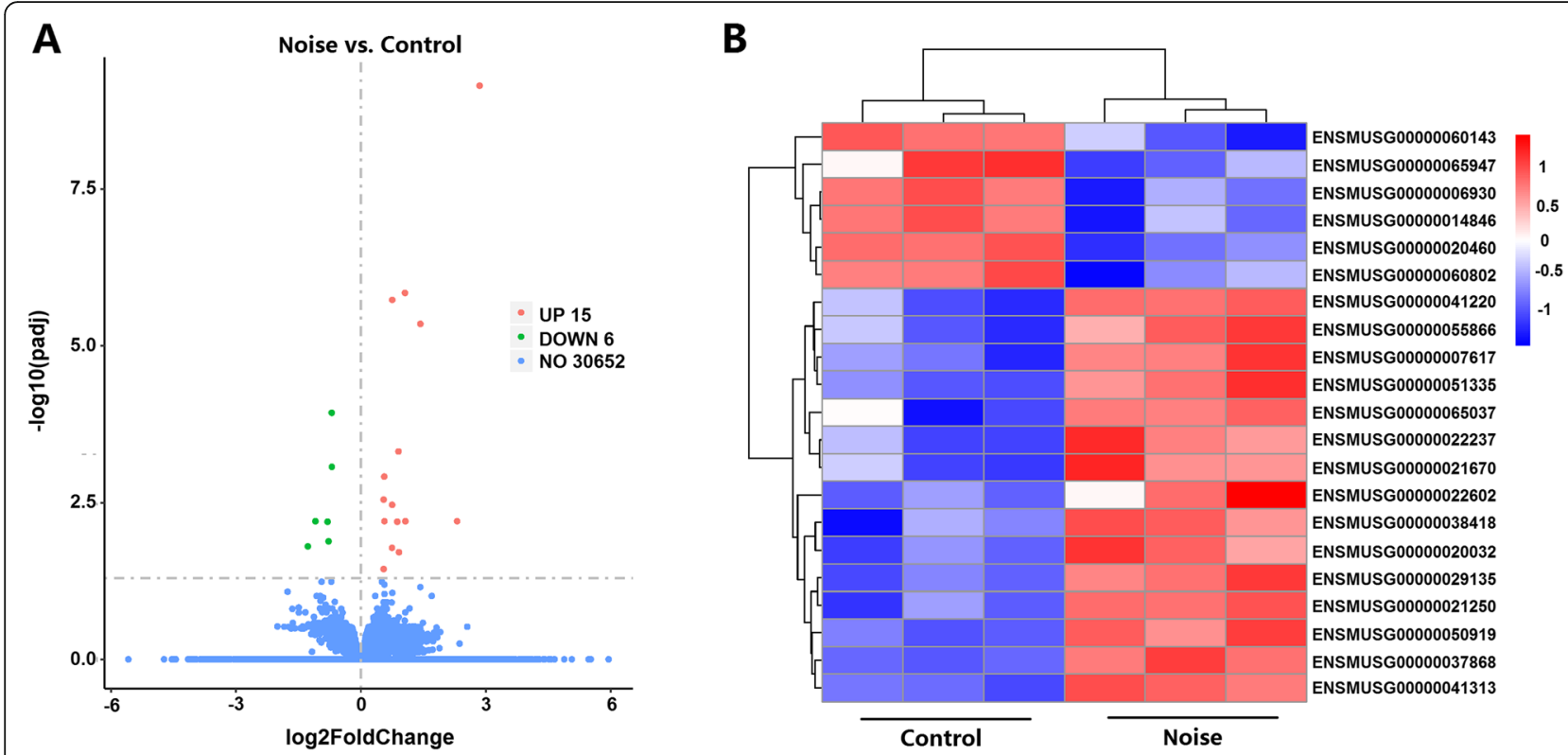

Fig. 2 Cluster analysis shows the differentially expressed mRNAs. a Differentially expressed mRNAs by volcano map. The red markings indicate upregulated expression, and the green markings indicate downregulated expression. b Differentially expressed mRNAs by heat map. The red markings indicate an upregulated expression, while the blue markings indicate a downregulated expression

involved in inflammatory reactions and apoptosis processes under conditions of oxidative stress [27]. It was also reported in our previous study that noise exposure could induce neural apoptosis [28], which is influenced by multiple factors and was shown to be closely related to the MAPK pathway [29]. AD is a multifactorial disease, and many theories have been formulated concerning its causes, including neuron loss, $\mathrm{A} \beta$ deposition, tau neuropathology, immune system dysfunction, synapse injury, oxidative stress, and mitochondrial dysfunction [30]. In $\mathrm{AD}$, the differential expression of genes can affect different signaling pathways [31]. As expected, our analysis showed that chronic noise exerted a multi-level effect on the acceleration of $\mathrm{AD}$ progress, which indicated that chronic noise exposure accelerated $\mathrm{AD}$ progress to a certain extent.

Table 5 AD-related genes identified after chronic noise exposure

\begin{tabular}{lll}
\hline Gene & Transcript ID & Foldchange \\
\hline Egr2 & ENSMUSG00000037868 & 7.25 \\
Fos & ENSMUSG00000021250 & 2.70 \\
Arc & ENSMUSG00000022602 & 1.89 \\
Egr1 & ENSMUSG00000038418 & 1.88 \\
Per2 & ENSMUSG00000055866 & 1.83 \\
Hmgcr & ENSMUSG00000021670 & 1.69 \\
Nauk1 & ENSMUSG00000020032 & 1.49 \\
Hap1 & ENSMUSG00000006930 & 0.62 \\
\hline
\end{tabular}

Our finding that AD-related genes [32-36], including Egr2, Fos, Arc, Hmgcr, Nuak1, Egr1, Hap1, Per2. Egr2, Fos, $A r c$, and $H m g c r$, show significant differential expression in noise-exposed mice lends support to the idea that environmental hazards including noise exposure may promote the occurrence and development of AD. Egr is a transcription factor and belongs to the family of immediate early genes induced by serum, which is closely related to the differentiation of some tissues and cells and is expressed in the early stage of central nervous system development, which promotes the formation of afterbrain. Upregulation of phagocytic markers Egr2 was observed in $\mathrm{A} \beta$ plaque-associated microglia, which reflects an attempt to enhance phagocytosis in plaque-associated microglia [37]. Egr-1 mRNA level is fourfold higher in AD brain than in non-AD brain, and the Egr-1 level is high in brain areas with high NFT density [38]. The overexpression of Egr-1 in rat brain could promote tau phosphorylation at Ser396/404 and Ser262 [39]. In the present study, we found an increase in the phosphorylation levels of tau protein at the Ser202 and Ser404 sites in the noise and aging groups. These alterations were also concomitant with the overexpression of Egr-1 after the end of the stimulus. Furthermore, lasting increase in Fos was found to occur in close correspondence with increase in tau hyperphosphorylation.

$A \beta$ precursor protein (APP) metabolism engages neuronal endolysosomal pathways for $A \beta$ processing and secretion. In AD, the dysregulation of APP leads to excess A $\beta$. Hap1 may promote the trafficking of APP into the 

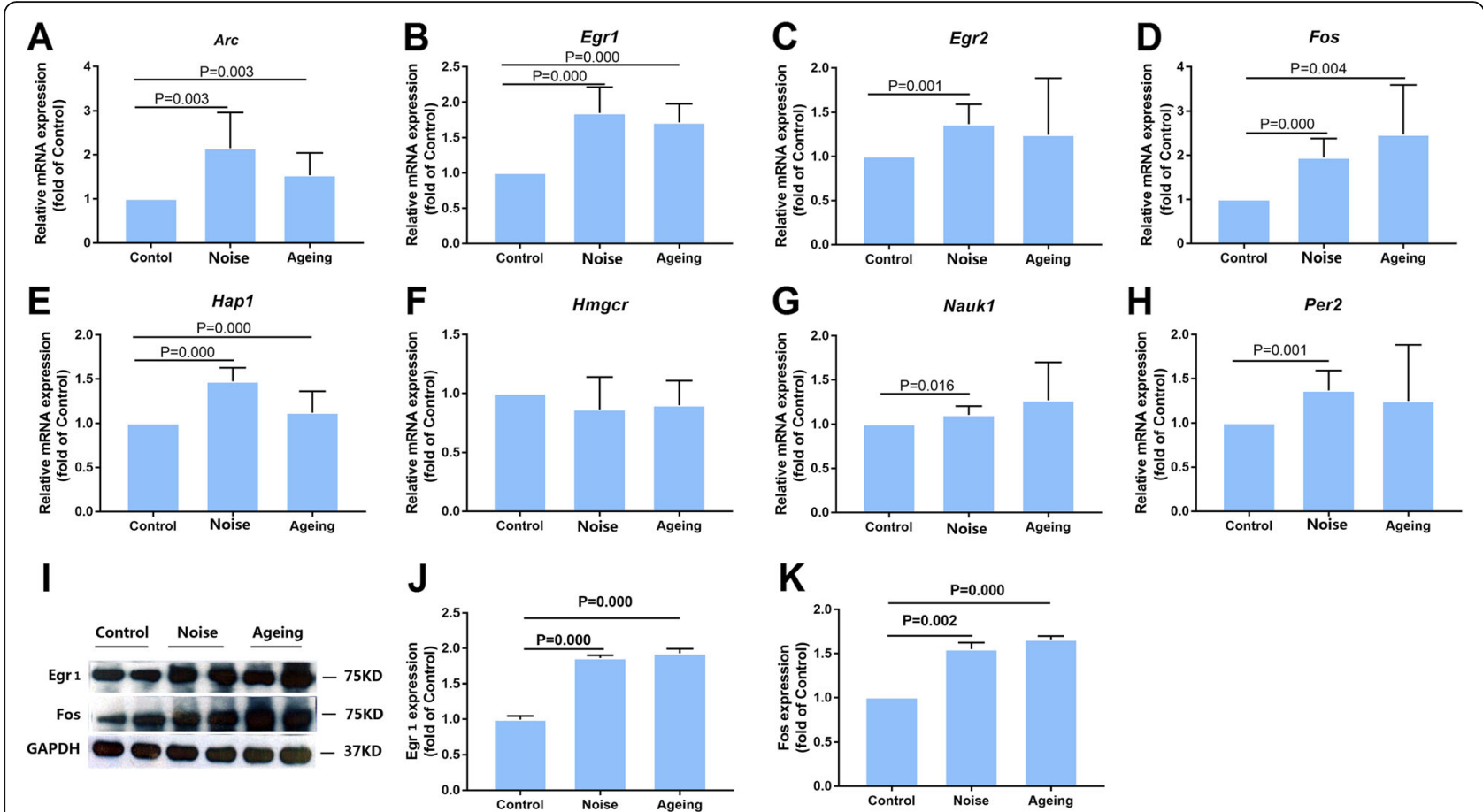

Fig. 3 Validation and quantitative assessment of selected AD-related differential genes. a-h. Validation of transcript expression by qPCR. mRNA expression levels of differential genes in SAMP8 mouse brain samples; GAPDH was used as a loading control. i-k. Quantitative assessment of Egr1 and Fos levels in the hippocampus. The density of the immunoreactive bands was quantified and represented as a percent change relative to the control; GAPDH was used as a loading control. Data were expressed as mean \pm standard deviation ( $n=6$ per group). Results were normalized with the control $=100 \%$

non-amyloidogenic pathway and reduce the production of $A \beta[40]$. In the present study, the amount of $A \beta$ increased when Hap1 was decreased; hence, regulating Hap1 expression may help to control $A \beta$ production and affect the development of AD. In our previous study, following noise stress, we detected significantly higher levels not only in $\mathrm{A} \beta$ but also in the phosphorylation of tau $[16,17,28,41]$. Our findings indicate that the long-lasting changes in tau phosphorylation observed after chronic noise exposure are likely to be the result of a complex regulatory network of multiple signaling pathways.

Due to the consideration of the influence of too many factors on the results of transcriptome, we did not use wild-type mice to set up the normal control group, which may affect the in-depth analysis of the research results. Additionally, the dose-response relationship of noise exposure was not considered in this study. More perfect experiments will be designed according to the important clues and limitations of the present study.

\section{Conclusion}

In summary, our results show the exacerbation of aginglike impairment following chronic noise exposure, as well as candidate genes mediating AD-like pathological changes in the hippocampus of noise-exposed SAMP8 mice. We also identify significant associations of genes with specific molecular pathway, which may be key candidate regulators involved in environment-gene interactions, and suggest that a more detailed look into the underlying mechanism of $\mathrm{AD}$ pathogenesis is warranted. Further detailed studies are required to clarify the molecular mechanisms underlying the regulation of the multifaceted signaling system in AD etiology.

\section{Supplementary information}

Supplementary information accompanies this paper at https://doi.org/10. 1186/s12199-019-0840-6.

Additional file 1: Figure $\mathbf{S 1}$. Sample overview of data characteristics. A The correlations between samples. $B$ The inter-group differences and the repetition of the samples within the group.

\section{Abbreviations}

AD: Alzheimer's disease; APP: A $\beta$ precursor protein; $A \beta$ : Amyloid beta; ELISA: Enzyme-linked immunosorbent assay; FPKM: Fragments per kilobase of transcript sequence per million; GO: Gene ontology; KEGG: Kyoto Encyclopedia of Genes and Genomes; NFTs: Neurofibrillary tangles; PCA: Principal component analysis; SAMP8: Senescence-accelerated mouse prone 8; SPL: Sound pressure level; TBST: Tris-buffered saline-Tween 


\section{Authors' contributions}

The text for this manuscript was written through the contribution of all authors. All authors have given approval to the final version of the manuscript.

\section{Funding}

Sources of Funding: This work was supported by grants from the National Natural Science Foundation of China (no. 81673136) and the National Natural Science Foundation of Tianjin (no. 17JCZDJC34900) awarded to B.C.

\section{Availability of data and materials}

All data generated or analyzed during this study are included in this published article.

\section{Ethics approval and consent to participate}

All animal handling procedures were reviewed and approved by the Animal Care/User Ethical Committee of Tianjin Institute of Environmental and Operational Medicine, Tianjin, China.

\section{Consent for publication}

All authors have approved the manuscript to be published.

\section{Competing interests}

The authors declare that they have no competing interests.

\section{Author details}

'Tianjin Institute of Environmental and Operational Medicine, Tianjin, China. ${ }^{2}$ School of Public Health and Management, Weifang Medical University, Weifang, China. ${ }^{3}$ Tianjin Centers for Disease Control and Prevention, Tianjin, China

Received: 28 August 2019 Accepted: 22 December 2019

Published online: 09 January 2020

\section{References}

1. Querfurth HW, LaFerla FM. Alzheimer's disease. N Engl J Med. 2010;362:329-44.

2. Cui B, Li K. Chronic noise exposure and Alzheimer disease: is there an etiological association? Med Hypotheses. 2013;81:623-6.

3. Gandy S. Perspective: prevention is better than cure. Nature. 2011;475:S15.

4. Sotiropoulos I, Cerqueira JJ, Catania C, Takashima A, Sousa N, Almeida OFX Stress and glucocorticoid footprints in the brain-the path from depression to Alzheimer's disease. Neurosci Biobehav Rev. 2008:32:1161-73.

5. Lupien SJ, Nair NPV, Briére S, Maheu F, Tu MT, Lemay M. Increased cortisol levels and impaired cognition in human aging: implication for depression and dementia in later life. Rev Neurosci. 1999;10:117-39.

6. Fjell AM, Mcevoy L, Holland D, Dale AM, Walhovd KB, Alzheimer's Disease Neuroimaging Initiative. What is normal in normal aging? Effects of aging, amyloid and Alzheimer's disease on the cerebral cortex and the hippocampus. Prog Neurobiol. 2014;117:20-40

7. Ma Q, Qiang J, Gu P, Wang Y, Geng Y, Wang M. Age-related autophagy alterations in the brain of senescence accelerated mouse prone 8(SAMP8) mice. Exp Gerontol. 2011;46:533-41.

8. Wyss-Coray T. Ageing, neurodegeneration and brain rejuvenation. Nature. 2016;539:180-6.

9. Elobeid A, Libard S, Leino M, Popova SN, Alafuzoff I. Altered proteins in the aging brain. J Neuropathol Exp Neurol. 2016;75:316-25.

10. Basner M, Babisch W, Davis A, Brink M, Clark C, Janssen S, Stansfeld S. Auditory and non-auditory effects of noise on health. Lancet. 2014; 383(9925):1325-32

11. Manikandan S, Padma MK, Srikumar R, Jeya Parthasarathy N, Muthuvel A, Sheela Devi R. Effects of chronic noise stress on spatial memory of rats in relation to neuronal dendritic alteration and free radicalimbalance in hippocampus and medial prefrontal cortex. Neurosci. Lett. 2006:399:17-22

12. Cui B, Zhu L, She X, Wu M, Ma Q, Wang T. Chronic noise exposure causes persistence of tau hyperphosphorylation and formation of NFT tau in the rat hippocampus and prefrontal cortex. Exp Neurol. 2012;238:122-9.
13. Cui B, Su D, Li W, She X, Zhang M, Wang R, Zhai Q. Effects of chronic noise exposure on the microbiome-gut-brain axis in senescence-accelerated prone mice: implications for Alzheimer's disease. J Neuroinflammation. 2018;15:190.

14. Gai Z, Su D, Wang Y, Li W, Cui B, Li K, She X, Wang R. Effects of chronic noise on the corticotropin-releasing factor system in the rat hippocampus: relevance to Alzheimer's disease-like tau hyperphosphorylation. Environ Health Prev Med. 2017;22:79.

15. Chen H, Kwong JC, Copes R, Tu K, Villeneuve PJ, Van DA, Hystad P, Martin RV, Muttay BJ, Jessiman B, Wilton AS, Kopp A, Burnett R. Living near major roads and the incidence of dementia, Parkinson's disease, and multiple sclerosis: a population-based cohort study. Lancet. 2017;389:718-26.

16. Clark C, Paunovic K. Who environmental noise guidelines for the European region: a systematic review on environmental noise and cognition. Int J Environ Res Public Health. 2018;15:285.

17. Zhang S, Zhu D, Li H, Li H, Feng C, Zhang W. Characterization of circRNAassociated-ceRNA networks in a senescence-accelerated mouse prone 8 brain. Mol. Ther. 2017:25:2053-61.

18. Kang L, Li S, Xing Z, Li J, Su Y, Fan P. Dihydrotestosterone treatment delays the conversion from mild cognitive impairment to Alzheimer's disease in SAMP8 mice. Horm Behav. 2014;65:505-15.

19. Li K, Jia H, She X, Cui B, Zhang N, Chen X, Xu C, An G, Ma Q. Role of NMDA receptors in noise-induced tau hyperphosphorylation in rat hippocampus and prefrontal cortex. J Neurol Sci. 2014;340:191-7.

20. Cui B, Li K, Gai Z, She X, Zhang N, Xu C. Chronic noise exposure acts cumulatively to exacerbate Alzheimer's disease-like Amyloid- $\beta$ pathology and neuroinflammation in the rat hippocampus. Sci Rep. 2015:5:12943.

21. Zhang S, Qin C, Cao G, Guo L, Feng C, Zhang W. Genome-wide analysis of DNA methylation profiles in a senescence-accelerated mouse prone 8 brain using whole-genome bisulfite sequencing. Bioinformatics. 2017;33:1591-5.

22. Wang $S$, Yu Y, Feng Y, Zou F, Zhang X, Huang J, Zhang Y, Zheng X, Huang $X F$, Zhu Y, Liu Y. Protective effect of the orientin on noise-induced cognitive impairments in mice. Behav Brain Res. 2015:296:290-300.

23. Cui B, Wu M, She $X$, Liu H. Impulse noise exposure in rats causes cognitive deficits and changes in hippocampal neurotransmitter signaling and tau phosphorylation. Brain Res. 2012;1427:35-43.

24. Ali F, Hossain MS, Sejimo S, Akashi K. Plasmalogens inhibit endocytosis of toll-like receptor 4 to attenuate the inflammatory signal in microglial cells. Mol Neurobiol. 2019:56(5):3404-19.

25. Tarkowski E, Liljeroth AM, Minthon L, Tarkowski A, Wallin A, Blennow K. Cerebral pattern of pro- and anti-inflammatory cytokines in dementias. Brain Res Bull. 2003;61:255-60

26. Sly LM, Krzesicki RF, Brashler JR, Buhl AE, McKinley DD, Carter DB, Chin JE. Endogenous brain cytokine mRNA and inflammatory responses to lipopolysaccharide are elevated in the Tg2576 transgenic mouse model of Alzheimer's disease. Brain Res Bull. 2001:56:581-8.

27. Ghasemi R, Zarifkar A, Rastegar K, Maghsoudi N, Moosavi M. Insulin protects against Abeta-induced spatial memory impairment, hippocampal apoptosis and MAPKs signaling disruption. Neuropharmacology. 2014;85:113-20.

28. Cui B, Wu MQ, Zhu LX, She XJ, Ma Q, Liu HT. Effect of chronic noise exposure on expression of $\mathrm{N}$-methyl-D-aspartic acid receptor $2 \mathrm{~B}$ and Tau phosphorylation in hippocampus of rats. Biomed Environ Sci. 2013:26:163-8.

29. Dickson DW. Apoptotic mechanisms in Alzheimer neurofibrillary degeneration: cause or effect? J Clin Invest. 2004:114:23-7.

30. Love JE, Hayden EJ, Rohn TT. Alternative splicing in Alzheimer's disease. J Parkinsons Dis Alzheimers Dis. 2015;2:6

31. Lu W, Mi R, Tang H, Liu S, Fan M, Wang L. Over-expression of c-fos mRNA in the hippocampal neurons in Alzheimer's disease. Chin Med J. 1998:111:35-7.

32. Zhang P, Hirsch EC, Damier P, Duyckaerts C, Javoy-Agid F. C-fos protein-like immunoreactivity: distribution in the human brain and over-expression in the hippocampus of patients with Alzheimer's disease. Neuroscience. 1992;46:9-21.

33. Morin JP, Cerón-Solano G, Velázquez-Campos G, Pacheco-López G, Bermúdez-Rattoni F, Díaz-Cintra S. Spatial memory impairment is associated with intraneural amyloid- $\beta$ immunoreactivity and dysfunctional arc expression in the hippocampal-CA3 region of a transgenic mouse model of Alzheimer's disease. J Alzheimers Dis. 2016;51:69-79.

34. von der Kammer H, Demiralay C, Andresen B, Albrecht C, Mayhaus M Nitsch RM. Regulation of gene expression by muscarinic acetylcholine receptors. Biochem Soc Symp. 2001;67:131-40.

35. Leduc $V$, Théroux L, Dea D, Dufour R, Poirer J. Effects of rs3846662 variants on HMGCR mRNA and protein levels and on markers of Alzheimer's disease pathology. J Mol Neurosci. 2016;58:109-19. 
36. Yin Z, Raj D, Saiepour N, Van Dam D, Brouwer N, Holtman IR, Eggen BJL, Möller T, Tamm JA, Abdourahman A, Hol EM, Kamphuis W, Bayer TA, De Deyn PP, Boddeke E. Immune hyperreactivity of A $\beta$ plaque-associated microglia in Alzheimer's disease. Neurobiol Aging. 2017:55:115-22.

37. Macgibbon GA, Lawlor PA, Walton M, Sirimanne E, Faull RLM, Synek B, Mee E, Connor B, Draqunow M. Expression of Fos, Jun, and Krox family proteins in Alzheimer's disease. Exp Neurol. 1997;147:316-32.

38. Lu Y, Li T, Qureshi HY, Han D, Paudel HK. Early growth response 1 (Egr-1) regulates phosphorylation of microtubule-associated protein tau in mammalian brain. J Biol Chem. 2011;286:20569-81.

39. Yang GZ, Yang M, Lim Y, Lu JJ, Wang TH, Qi JG, Zhong JH, Zhou XF. Huntingtin associated protein 1 regulates trafficking of the amyloid precursor protein and modulates amyloid beta levels in neurons. J Neurochem. 2012;122:1010-22.

40. Gai Z, Li K, Sun H, She X, Cui B, Wang R. Effects of chronic noise on mRNA and protein expression of CRF family molecules and its relationship with $\mathrm{p}$ tau in the rat prefrontal cortex. J Neurol Sci. 2016;368:307-13.

\section{Publisher's Note}

Springer Nature remains neutral with regard to jurisdictional claims in published maps and institutional affiliations.

Ready to submit your research? Choose BMC and benefit from:

- fast, convenient online submission

- thorough peer review by experienced researchers in your field

- rapid publication on acceptance

- support for research data, including large and complex data types

- gold Open Access which fosters wider collaboration and increased citations

- maximum visibility for your research: over $100 \mathrm{M}$ website views per year

At $\mathrm{BMC}$, research is always in progress.

Learn more biomedcentral.com/submissions 